\title{
High-spin isomers: structure and applications
}

\author{
P.M. Walker ${ }^{\mathrm{a}}$
}

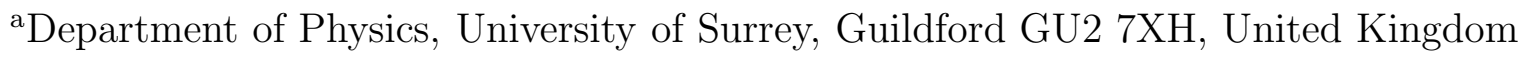

High-spin-isomer research is briefly reviewed, with attention to the highest spins, the heaviest elements, proton-rich nuclei and neutron-rich nuclei. The utility of isomers is emphasised in connection with the study of exotic nuclei, where isomeric states may be longer lived than ground states. It is pointed out that isomers may also provide opportunities for studying effects at the atomic-nuclear interface.

\section{INTRODUCTION}

The year 2009 marks the $100^{\text {th }}$ anniversary of the first nucleus-nucleus collisions, $\alpha$ particles on gold and other elements, studied by Geiger and Marsden [1]. Today, beams of all stable nuclides, and many radioactive ones, can be produced. Even some purified isomeric beams are now a reality [2] or under development [3]. While several different themes of isomer physics have been discussed in earlier topical reviews [4,5], the present paper addresses some recent results and future opportunities with isomers at high-spin and in exotic nuclei.

Isomers are excited, metastable states of nuclei, with long half-lives. Even though the half-life limit is not well defined, it is useful to consider that isomers should live for a least a few nanoseconds, which is several orders of magnitude longer than typical excitedstate half-lives. This gives sufficient time to perform selective experiments, by separating the bulk of the radiation from that associated with isomers, following their formation in nuclear collisions.

The key physical quantity that leads to isomerism is angular momentum. If the internal $\gamma$-ray and electron-conversion emission from a nuclear excited state is forced, on account of the proton and neutron orbits involved, to proceed through a high-angular-momentum transition, then the decay is slow and an isomer results. Such an isomer may be called a "spin trap". Furthermore, a deformed nucleus with an axis of symmetry has approximate conservation of the symmetry-axis projection of the angular momentum, designated " $K$ ", and large changes in this quantity result in " $K$ isomers". Finally, there can also be "shape isomers", where it is a shape change that inhibits decay, rather than angular momentum. Longer-lived isomers sometimes result from a combination of these mechanisms.

Although it is possible to view the occurrence of isomers as arising from somewhat chance couplings of particular nucleon orbits, such couplings produce unusual states, typically with well defined wave functions, which can be directly related to shell-model configurations. These states have been key to the early development and understanding

*With support from UK STFC and AWE plc. 
of the shell model [6], and the close relationship with the shell model continues today, as we explore the persistence (or otherwise) of shell gaps in exotic nuclei [7].

\section{ISOMERS AT THE HIGHEST SPINS}

One of the important physical features at high excitation energy and/or high angular momentum is the loss of pairing correlations. In this regard, rotational bands associated with high-spin isomers provide a unique perspective: broken pair configurations block the pairing, and the rotational-band moments of inertia show the consequences. This can be illustrated by the $K=30,8$-quasiparticle rotational band in ${ }^{178} \mathrm{~W}$ [8], as shown in Fig. 1. Compared to the rigid-body value, the moment of inertia of the $K=30$ band is reduced by about one third, and tilted-axis-cranking (TAC) calculations [9] show that this reduction is compatible with completely quenched pairing effects (although dynamical pairing correlations remain [10]). It should be added that the selection of this $K=30$ band is judiciously based on its configuration, and the lack of high- $j$, low- $\Omega$ quasiparticles, which can align their angular momentum with the collective rotation and considerably complicate the situation. It would be highly desirable to confirm this moment-of-inertia effect, but no other existing data are directly comparable. The highest-seniority isomer recorded to date is the $K=57 / 2,9$-quasiparticle isomer in ${ }^{175} \mathrm{Hf}$ [11], but the associated rotational bands [12] remain to be characterised.

The highest-spin isomers that have been observed are in spherical (or near-spherical) nuclei. Spin $I \approx 38 \hbar$ has been reached in ${ }^{212} \mathrm{Rn}[13]$, involving triple neutron core excitations. Here, one of the general physics issues is whether or not significant deformation develops at high angular momentum, but, so far, more-detailed model calculations are required in order to understand the complex experimental level properties.

It could be supposed that at very high spin isomers might no longer exist, due to deformation and rotation-alignment effects. However, there is no clear evidence for this. In contrast, it can be argued that the most favourable cases (such as in the stable and neutron-rich hafnium isotopes [4]) are simply not accessible at very high spin, due to the unavailability of stable beam and target combinations that provide sufficient angular momentum from fusion-evaporation reactions. The next generation of radioactive beams can be expected to overcome this restriction, although the required sensitivity may be difficult to achieve in the presence of background radioactivity from the beam particles.

\section{ISOMERS IN THE HEAVIEST NUCLEI}

In some of the heaviest nuclei, isomeric states can be longer lived than their respective ground states [14], and this may be important for future experimental investigations of the upper mass limit of nuclear binding. Despite the fact that there is little systematic knowledge about $K$-forbidden transition rates in such nuclei, comparisons with lower mass regions can be instructive. Furthermore, there has been recent progress in the study of high-spin isomers in $Z \approx 100$ nuclides [15], though knowledge of isomeric decay transitions is so far restricted to those from one- and two-quasiparticle states.

Fig. 2 shows how the $K$-forbidden $E 1$ decay rate from the $K^{\pi}=8^{-}$isomer in ${ }^{252}$ No [16] compares with corresponding transition rates in hafnium $(Z=72)$ isotopes. The horizontal axis is the ratio of the ground-state-band dynamic and kinematic moments of 


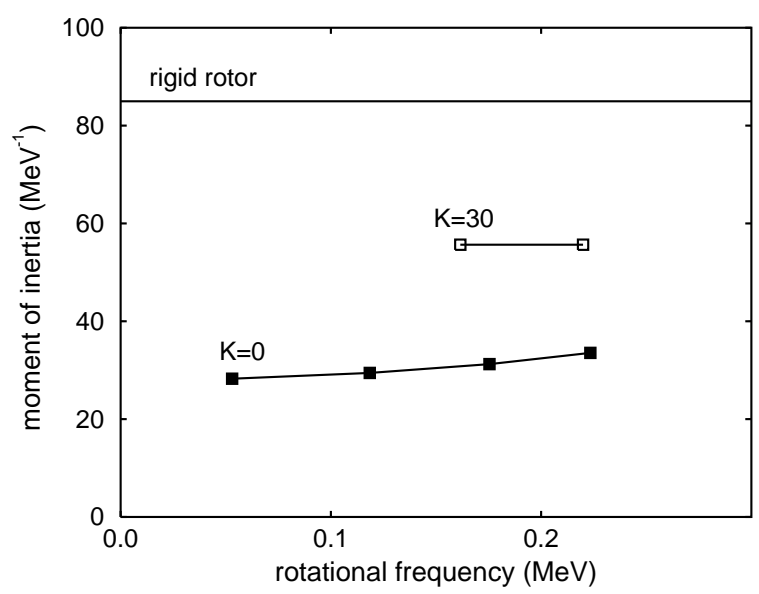

Figure 1. Kinematic moments of inertia as a function of rotational frequency for the first few states of the $K=0$, ground-state band of ${ }^{178} \mathrm{~W}$, and for the $K=30$, 8-quasiparticle band, compared with the rigid-body value.

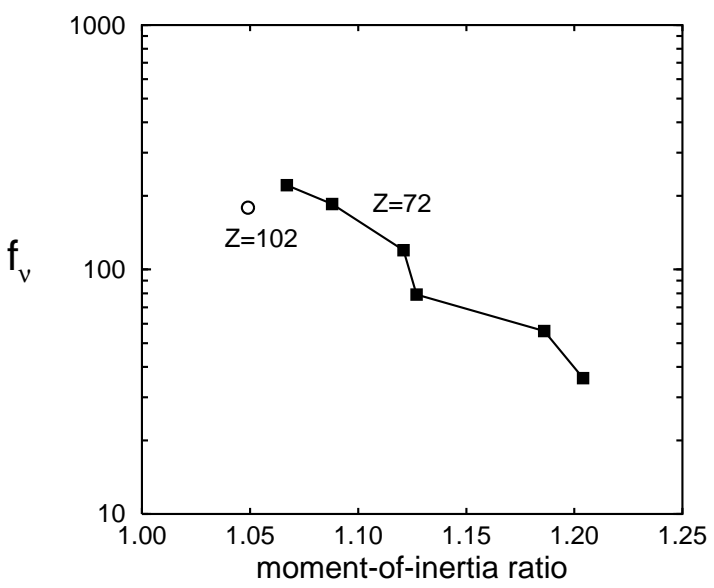

Figure 2. Reduced hindrance values for $\Delta K=8, E 1$ transitions from two-quasiparticle isomers in even-even hafnium isotopes (filled squares) and ${ }^{252}$ No (open circle). The horizontal axis is the ratio of the ground-state-band dynamic and kinematic moments of inertia.

inertia, which gives a measure of the Coriolis-induced rotational-alignment effects, i.e. it reflects the degree of $K$-mixing. The strong inverse correlation, already known for the $A \approx 180$ hafnium isotopes [17], is seen to extend to the $Z \approx 100$ region, where the nuclear rigidity leads to very large $K$ hindrance. The comparatively large $K$ hindrance also applies to $K$-forbidden $E 2$ transitions, shown in Fig. 3 as a function of the product of the valence nucleon numbers $\left(\mathrm{N}_{p} \mathrm{~N}_{n}\right)$. This extends the analysis of Refs $[18,19]$. It is apparent that $K$-forbidden transitions in some of the heaviest elements are amongst the most hindered of any nuclides, supporting the viewpoint the $K$ quantum number has a vital role to play in their structure and survival.

\section{ISOMERS IN PROTON-RICH NUCLEI}

A proton can "drip" from an isomer before the proton dripline itself is reached, simply on account of the isomer's excitation energy. Indeed, a high-spin $\left(19 / 2^{-}\right)$isomer in ${ }^{53} \mathrm{Co}$ provided the first example of proton radioactivity [20]. The recent observation of proton decay from a $10^{+}$isomer in ${ }^{54} \mathrm{Ni}[21]$ is of particular interest from an experimental point of view, because the proton emission was deduced without observing any protons, but instead through the observation of delayed $\gamma$-rays at a spectrometer setting which dictated that a proton must have been emitted. It can be supposed that neutron radioactivity from a high-spin isomer might one day be detected in a corresponding manner, with the isomerism being due to the angular-momentum barrier [22].

A remarkable proton-decaying case is the $21^{+}$isomer in ${ }^{94} \mathrm{Ag}$, at over $6 \mathrm{MeV}$ excitation 


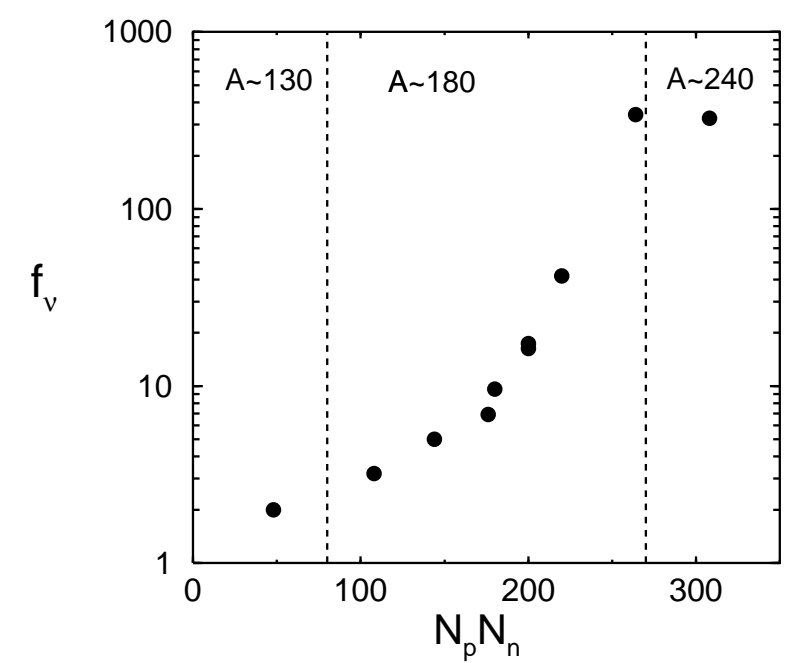

Figure 3. Reduced hindrance values for $\Delta K=6$ and 10, E2 transitions from twoquasiparticle isomers in even-even nuclides, as a function of the product, $\mathrm{N}_{p} \mathrm{~N}_{n}$, of the valence nucleon numbers. The data for the lightest and heaviest cases refer to isomers in ${ }^{134} \mathrm{Ce}$ and ${ }^{244} \mathrm{Cm}$, respectively.

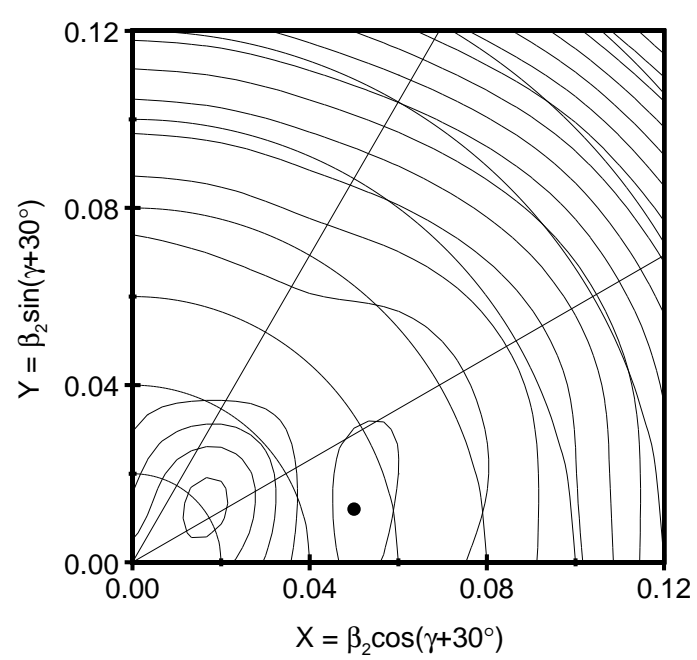

Figure 4. Configuration constrained potential-energy surface for the ${ }^{94} \mathrm{Ag}, 21^{+}$ isomer. The minimum, indicated by a dot, is at $\beta_{2}=0.05$. Contours are at $200 \mathrm{keV}$ intervals.

energy [23], which lives ten times as long as its respective ground state. The reported observation of two-proton emission from the isomer [24] seems to require a highly elongated nuclear shape. However, there are problems with this interpretation, not least because shell-model calculations [25] indicate near-spherical shape for that spin and parity. Even starting from a deformed Woods-Saxon basis, the configuration-constrained [26] potential-energy surface shows little deformation, as illustrated in Fig. 4 [27], where the $\nu\{5 / 2[422], 7 / 2[413], 9 / 2[404]\} \otimes \pi\{5 / 2[422], 7 / 2[413], 9 / 2[404]\} 21^{+}$configuration has a calculated excitation energy of $5.16 \mathrm{MeV}$. Further work is needed to clarify the nature of this exceptional isomer.

\section{ISOMERS IN NEUTRON-RICH NUCLEI}

As noted in the previous section, there could be neutron-decaying isomers close to the neutron dripline, but so far experiment seems to be far from that frontier. Nevertheless, important information relevant to the rapid-neutron-capture ( $r$-process) nucleosynthesis pathway can already be obtained from isomers [7]. There are also several nuclear-structure predictions for long-lived isomers in neutron-rich nuclides, such as ${ }^{188} \mathrm{Hf}$ and ${ }^{170} \mathrm{Dy}[28,29]$. These present significant experimental challenges, not least because half-lives greater than $1 \mathrm{~s}$ are too long for delayed-coincidence techniques that correlate individual ions with their isomeric $\gamma$-ray decays. However, for such long half-lives, it becomes possible to 
use high-resolution mass spectrometry. For example, a Penning trap at NSCL has been successfully used to identify a long-lived (>150 ms) isomer in ${ }^{65} \mathrm{Fe}$ [30]. Furthermore, with the Experimental Storage Ring (ESR) at GSI, a long-lived ( $>1 \mathrm{~s}$ ) isomer was identified in ${ }^{125} \mathrm{Ce}$ [31], and new data are under analysis which promise to open up the neutron-rich domain for the study of long-lived high-spin isomers.

\section{ISOMER APPLICATIONS}

Some of the work discussed above can be described as "applications" of isomers, in the sense that it can be the states populated in isomer decays that are the focus of interest, rather than the isomers themselves. Isomers can thus be considered as tools that may be applied selectively to probe the structure of exotic nuclei, often with an astrophysical interest [32].

Wider ranging applications of isomers are also a possibility $[5,32,33]$. The controlled release of stored isomer energy has been one focus of attention. Although this has not yet been convincingly achieved with low-energy photons [34], the coupling of atomic-electrons to the nucleus may offer an alternative mechanism. In particular, the process of nuclear excitation by electron capture (NEEC) might be exploited $[35,36]$. Indeed, since NEEC itself has never been measured experimentally, it could be that isomers will provide the means to observe this basic process for the first time.

\section{SUMMARY}

Isomers are deeply imbedded in the disciplines of nuclear structure and nuclear astrophysics. Due to their long half-lives, isomers can be used to "parachute" into regions of exotic nuclei, and thus to reveal features that would otherwise remain obscured. Furthermore, there are potential applications of isomers at the interface between atomic and nuclear physics, associated with the electronic manipulation of nuclear excitations. The advent of radioactive beams, including purified isomeric beams, now provides exceptional discovery potential.

\section{REFERENCES}

1. H. Geiger and E. Marsden, Proc. Roy. Soc. 82 (1909) 495.

2. I. Stefanescu et al., Phys. Rev. Lett. 98 (2007) 122701.

3. H. Geissel et al., Eur. Phys. J. Special Topics 150 (2007) 109.

4. P.M. Walker and G.D. Dracoulis, Nature 399 (1999) 35.

5. P.M. Walker and J.J. Carroll, Nucl. Phys. News 17, No. 2 (2007) 11.

6. E. Segrè and A.C. Helmholz, Rev. Mod. Phys. 21 (1949) 271.

7. A. Jungclaus et al., Phys. Rev. Lett. 99 (2007) 132501.

8. D.M. Cullen et al., Phys. Rev. C60 (1999) 064301.

9. S. Frauendorf, K. Neergård, J.A. Sheikh and P.M. Walker, Phys. Rev. C61 (2000) 064324.

10. D. Almehed, S. Frauendorf and F. Dönau, Phys. Rev. C63 (2001) 044311.

11. N.L. Gjørup, M.A. Bentley, B. Fabricius, A. Holm, J.F. Sharpey-Schafer, G. Sletten and P.M. Walker, Z. Phys. A337 (1990) 353. 
12. F.G. Kondev et al., to be published.

13. G.D. Dracoulis, G.J. Lane, A.P. Byrne, P.M. Davidson, T. Kibédi, P. Nieminen, H. Watanabe and A.N. Wilson, Phys. Lett. B662 (2008) 19.

14. F.R. Xu, E.G. Zhao, R. Wyss and P.M. Walker, Phys. Rev. Lett. 92 (2004) 252501.

15. R.D. Herzberg and P.T. Greenlees, Prog. Part. Nucl. Phys. 61 (2008) 647.

16. B. Sulignano et al., Eur. Phys. J. A33 (2007) 327.

17. P.M. Walker, G.D. Dracoulis, A.P. Byrne, T. Kibédi and A.E. Stuchbery, Phys. Rev. C49 (1994) 1718.

18. P.M. Walker, J. Phys. G16 (1990) L233.

19. P.M. Walker and K. Schiffer, Z. Phys. A338 (1991) 239.

20. K.P. Jackson, C.U. Cardinal, H.C. Evans, N.A. Jelley and J. Cerny, Phys. Lett. B33 (1970) 281.

21. D. Rudolph et al., Phys. Rev. C78 (2008) 021301(R).

22. L.K. Peker, E.I. Volmyansky, V.E. Bunakov and S.G. Ogloblin, Phys. Lett. B36 (1971) 547.

23. A. Kankainen et al., Phys. Rev. Lett. 101 (2008) 142503.

24. I. Mukha et al., Nature 439 (2006) 298.

25. K. Kaneko, Y. Sun, M. Hasegawa and T. Mizusaki, Phys. Rev. C77 (2008) 064304; C78 (2008) 049903(E).

26. F.R. Xu, P.M. Walker, J.A. Sheikh and R. Wyss, Phys. Lett. B435 (1998) 257.

27. P.M. Walker, F.R. Xu and H.L. Liu, to be published.

28. P.M. Walker and G.D. Dracoulis, Hyp. Int. 135 (2001) 83.

29. P.H. Regan et al., Phys. Rev. C65 (2002) 037302.

30. M. Block et al., Phys. Rev. Lett. 100 (2008) 132501.

31. B. Sun et al., Eur. Phys. J. A31 (2007) 393.

32. A. Aprahamian and Y. Sun, Nature Phys. 1 (2005) 81.

33. G.C. Baldwin and J.C. Solem, Rev. Mod. Phys. 69 (1997) 1085.

34. J.J. Carroll et al., Phys. Lett. B679 (2009) 203.

35. G. Gosselin, V. Méot and P. Morel, Phys. Rev. C76 (2007) 044611.

36. A. Pálffy et al., Phys. Lett. B661 (2008) 330. 